

\title{
CIRCULANT MATRICES AND TIME-SERIES ANALYSIS
}

\author{
by D.S.G. Pollock \\ Queen Mary, University of London \\ and \\ GREQAM: Groupement de Recherche en \\ Economie Quantitative d'Aix-Marseille \\ Email: stephen_pollock@sigmapi.u-net.com \\ This paper sets forth some of the salient results in the algebra of circu- \\ lant matrices which can be used in time-series analysis. It provides easy \\ derivations of some results that are central to the analysis of statistical pe- \\ riodograms and empirical spectral density functions. A statistical test for \\ the stationarity or homogeneity of empirical processes is also presented.
}

Date: October 2000.

Key words: Time-series analysis, Circulant matrices, Discrete Fourier transforms, Periodograms;

JEL Classification: C22.

\section{Introduction}

A real circulant stochastic process of order $T$ is one in which the autocovariance matrix of a vector $\left[y_{0}, y_{1}, \ldots, y_{T-1}\right]$, sampled from the process, is unchanged when the elements of the vector are subjected to a cyclical permutation. Thus, for any integers $s, t \in[0, T-1]$, we should have

$$
\gamma_{|t-s|}=C\left(y_{t}, y_{s}\right)=C\left\{y_{(t+\tau \bmod T)}, y_{(s+\tau \bmod T)}\right\}
$$

where $\tau$ is an arbitrary integer. Such a process is the finite equivalent of a stationary stochastic process.

An ordinary stationary process is, by definition, distributed over the set of all positive and negative integers, which corresponds to a set of equally spaced points on the time axis. It is statistically invariant with respect to translations along this axis. A circular process, in comparison, is invariant with respect of translations around a closed circuit where the successive stations are indexed by the integers $0,1, \ldots, T-1$.

One of the bugbears of time-series analysis is the need to cope with the fact that all data series are finite with a definite beginning and an end. Often we need to infer the values of elements that lie outside the sample. Also, in attempting to determine the sampling properties of time-series estimates, we have often to contend with the disjunctions at the beginning and the end of the samples. Usually we can show that, as the sample size increases, the effect 


\section{D.S.G. POLLOCK: Circulant Matrices}

of these disjunctions upon our estimates vanishes; but it is often difficult or laborious to demonstrate the fact.

A virtue of circular processes is that they suffer from none of these end effects. They are often easier to analyse than are the corresponding finite segments sampled from stationary processes, and the results that are obtained are usually perfect prototypes for the asymptotic results for stationary processes.

The theoretical importance of circular processes is reaffirmed once it is recognised that all time-series methods that make use of the discrete Fourier transform are effectively based upon the assumption that the data are from circular processes.

The purpose of this paper is to set forth some of the salient results in the algebra of circular processes which can be used in time-series analysis. In the course of doing so, we shall provide easy proofs of some results that are central to the analysis of statistical periodograms and empirical spectral density functions. We shall also derive a statistical test for the stationarity or homogeneity of an empirical process. Once the algebra of circulant matrices has been digested, it should appear that the test is of a familiar classical nature.

Circulant matrices have represented a mathematical curiosity ever since their first appearance in the literature in a paper by Catalan [2]. The literature on circulant matrices, from their introduction until 1920, was summarised in four papers by Muir [6]-[9]. A recent treatise on the subject, which contains a useful bibliography, has been provided by Davis [4]; but his book does not deal with problems in time-series analysis.

Circulant matrices have been used, in passing, in the analysis of time series by several authors, notably by Anderson [1] and by Fuller [5], but their usefulness in organising the material of statistical Fourier analysis, or of timeseries analysis in the frequency domain, has not been fully exploited. It is hoped that the paper will help in changing this.

\section{Circulant Matrices and Polynomials}

A circulant matrix is a Toeplitz matrix which has the general form of

$$
A=\left[\begin{array}{ccccc}
\alpha_{0} & \alpha_{T-1} & \alpha_{T-2} & \ldots & \alpha_{1} \\
\alpha_{1} & \alpha_{0} & \alpha_{T-1} & \ldots & \alpha_{2} \\
\alpha_{2} & \alpha_{1} & \alpha_{0} & \ldots & \alpha_{3} \\
\vdots & \vdots & \vdots & \ddots & \vdots \\
\alpha_{T-1} & \alpha_{T-2} & \alpha_{T-3} & \ldots & \alpha_{0}
\end{array}\right]
$$

The vectors of such a matrix are generated by applying a succession of cyclic permutations to the leading vector, which therefore serves to specify the matrix completely. The elements of the circulant matrix $A=\left[\alpha_{i j}\right]$ fulfil the condition that $\alpha_{i j}=\alpha\{(i-j) \bmod T\}$. Hence, the index for the supra-diagonal elements, for which $1-T<(i-j)<0$, becomes $(i-j) \bmod T=T+(i-j)$.

The operator which effects the cyclic permutation of the elements of any (column) vector of order $T$ is the matrix $K=\left[e_{1}, \ldots, e_{T-1}, e_{0}\right]$. This is formed 


\section{D.S.G. POLLOCK: Circulant Matrices}

from the identity matrix $I=\left[e_{0}, e_{1}, \ldots, e_{T-1}\right]$ by moving the leading vector to the back of the array. Thus

$$
K=\left[\begin{array}{cccccc}
0 & 0 & 0 & \ldots & 0 & 1 \\
1 & 0 & 0 & \ldots & 0 & 0 \\
0 & 1 & 0 & \ldots & 0 & 0 \\
\vdots & \vdots & \vdots & \ddots & \vdots & \vdots \\
0 & 0 & 0 & \ldots & 0 & 0 \\
0 & 0 & 0 & \ldots & 1 & 0
\end{array}\right]
$$

We should observe that the following conditions hold:

$$
\begin{array}{ll}
\text { (i) } & K^{-q}=K^{T-q}, \\
\text { (ii) } & K^{0}=K^{T}=I, \\
\text { (iii) } & K^{\prime}=K^{T-1}=K^{-1} .
\end{array}
$$

Any circulant matrix $A$ of order $T$ can be expressed as a linear combination of the set of basis matrices $I, K, \ldots, K^{T-1}$; and thus $A$ can be expressed as a polynomial function

$$
\begin{aligned}
A & =\alpha_{0} I+\alpha_{1} K+\cdots+\alpha_{T-1} K^{T-1} \\
& =\alpha(K)
\end{aligned}
$$

If $\left\{\gamma_{i}\right\}$ is an absolutely convergent sequence obeying the condition that $\sum\left|\gamma_{i}\right|<\infty$, then the $z$-transform of the sequence, which is defined by $\gamma(z)=$ $\sum \gamma_{j} z^{j}$, is an analytic function on the unit circle. In that case, replacing $z$ by $K$ gives rise to a circulant matrix $\Gamma=\gamma(K)$ with finite-valued elements. Noting that $K \uparrow q=K \uparrow(q \bmod T)$, it is found that

$$
\begin{aligned}
\Gamma & =\left\{\sum_{j=0}^{\infty} \gamma_{j T}\right\} I+\left\{\sum_{j=0}^{\infty} \gamma_{(j T+1)}\right\} K+\cdots+\left\{\sum_{j=0}^{\infty} \gamma_{(j T+T-1)}\right\} K^{T-1} \\
& =\psi_{0} I+\psi_{1} K+\cdots+\psi_{T-1} K^{T-1}
\end{aligned}
$$

Given that $\left\{\gamma_{i}\right\}$ is a convergent sequence, it follows that the sequence of the matrix coefficients $\left\{\psi_{0}, \psi_{1}, \ldots, \psi_{T-1}\right\}$ converges to $\left\{\gamma_{0}, \gamma_{1}, \ldots, \gamma_{T-1}\right\}$ as $T$ increases.

Notice that the matrix $\psi(K)=\psi_{0} I+\psi_{1} K+\cdots+\psi_{T-1} K^{T-1}$, which is derived from a polynomial $\psi(z)$ of degree $T-1$, is a synonym for the matrix $\gamma(K)$, which is derived from the $z$-transform of an infinite convergent sequence.

The polynomial representation is enough to establish that circulant matrices commute in multiplication and that their product is also a polynomial in $K$. That is to say

If $A=\alpha(K)$ and $B=\beta(K)$ are circulant matrices, then their product $\Gamma=A B=B A$ is also a circulant matrix. 


\section{D.S.G. POLLOCK: Circulant Matrices}

The peculiar feature of such polynomials is that the powers of the argument $K$ form a $T$-periodic sequence such that $K^{j+T}=K^{j}$; and thus the degrees of the polynomial products never exceed $T-1$. This periodicity is analogous to the periodicity of the argument $z=\exp \{-i 2 \pi / T\}$ which is to be found in the Fourier transform of a sequence of order $T$.

Notice also that the characterisation of $A=\alpha(K)$ under (5) implies that

$$
K A K^{\prime}=K^{\prime} A K=A .
$$

A symmetric circulant matrix $C=C^{\prime}$ must have a representation in the form of

$$
\begin{aligned}
C & =\gamma_{0} I+\gamma_{1}\left(K+K^{-1}\right)+\cdots+\gamma_{T-1}\left(K^{T-1}+K^{1-T}\right) \\
& =\gamma_{0} I+\left(\gamma_{1}+\gamma_{T-1}\right) K+\cdots+\left(\gamma_{T-1}+\gamma_{1}\right) K^{T-1} .
\end{aligned}
$$

The implication of the condition is that $c_{\tau}=\left(\gamma_{\tau}+\gamma_{T-\tau}\right)=c_{T-\tau}$.

Observe that this condition of matrix symmetry can be obtained directly from the defining conditions of a circulant matrix. For, if $\alpha_{i j}=\alpha\{(i-j) \bmod$ $T\}$ is the element in the $i$ th row and the $j$ th column and if $\alpha_{i j}=\alpha_{j i}$, then $\alpha\{\tau \bmod T\}=\alpha\{-\tau \bmod T\}$ where $\tau=i-j$, which is to say that $\alpha_{\tau}=\alpha_{T-\tau}$.

An example of a symmetric circulant matrix is provided by the matrix of the autocovariances of a circular process. If the sequence $\left\{\gamma_{0}, \gamma_{1}, \ldots, \gamma_{T-1}\right\}$ represents a sequence of ordinary autocovariances, and if $\gamma_{\tau}=0$ for $\tau \geq T$, then $\left\{c_{0}=\gamma_{0}, c_{1}=\left(\gamma_{1}+\gamma_{T-1}\right), \ldots, c_{T-1}=\left(\gamma_{T-1}+\gamma_{1}\right)\right\}$ would be the corresponding sequence of circular autocovariances.

Example. Imagine that $T$ observations, running from $t=0$ to $t=T-1$, have been taken on a stationary and invertible $\operatorname{ARMA}(p, q)$ process $y(t)$ which is described by the equation

$$
\left(1+\alpha_{1} L+\cdots+\alpha_{p} L^{p}\right) y(t)=\left(1+\mu_{1} L+\cdots+\mu_{q} L^{q}\right) \varepsilon(t),
$$

wherein $\varepsilon(t)$ is a white-noise sequence of independently and identically distributed random variables of zero mean, and $L$ is the lag operator which has the effect that $L \varepsilon(t)=\varepsilon(t-1)$. Corresponding to the observations, there is a set of $T$ equations which can be arrayed in a matrix format:

$$
\left[\begin{array}{cccc}
y_{0} & y_{-1} & \ldots & y_{-p} \\
y_{1} & y_{0} & \ldots & y_{1-p} \\
\vdots & \vdots & \ddots & \vdots \\
y_{p} & y_{p-1} & \ldots & y_{0} \\
\vdots & \vdots & \ddots & \vdots \\
y_{T-1} & y_{T-2} & \ldots & y_{T-p-1}
\end{array}\right]\left[\begin{array}{c}
1 \\
\alpha_{1} \\
\vdots \\
\alpha_{p}
\end{array}\right]=\left[\begin{array}{cccc}
\varepsilon_{0} & \varepsilon_{-1} & \ldots & \varepsilon_{-q} \\
\varepsilon_{1} & \varepsilon_{0} & \ldots & \varepsilon_{1-q} \\
\vdots & \vdots & \ddots & \vdots \\
\varepsilon_{q} & \varepsilon_{q-1} & \ldots & \varepsilon_{0} \\
\vdots & \vdots & \ddots & \vdots \\
\varepsilon_{T-1} & \varepsilon_{T-2} & \ldots & \varepsilon_{T-q-1}
\end{array}\right]\left[\begin{array}{c}
1 \\
\mu_{1} \\
\vdots \\
\mu_{q}
\end{array}\right] .
$$

Here, the generic equation is

$$
\sum_{i=0}^{p} \alpha_{i} y_{t-i}=\sum_{i=0}^{q} \mu_{i} \varepsilon_{t-i}, \quad \text { where } \quad \alpha_{0}=\mu_{0}=1 .
$$


Apart from the elements $y_{0}, y_{1}, \ldots, y_{T-1}$ and $\varepsilon_{0}, \varepsilon_{1}, \ldots, \varepsilon_{T-1}$ which fall within the sample period, these equations comprise the presample values $y_{-p}, \ldots, y_{-1}$ and $\varepsilon_{-q}, \ldots, \varepsilon_{-1}$ which are to be found in the top-right corners of the matrices.

An alternative representation of the system of equations can be given which is in terms of polynomials. Thus, if

$$
\begin{aligned}
& y(z)=y_{-p} z^{-p}+\cdots+y_{0}+y_{1} z+\cdots+y_{T-1} z^{T-1}, \\
& \varepsilon(z)=\varepsilon_{-q} z^{-q}+\cdots+\varepsilon_{0}+\varepsilon_{1} z+\cdots+\varepsilon_{T-1} z^{T-1}, \\
& \alpha(z)=1+\alpha_{1} z+\cdots+\alpha_{p} z^{p} \quad \text { and } \\
& \mu(z)=1+\mu_{1} z+\cdots+\mu_{q} z^{q}
\end{aligned}
$$

then

$$
y(z) \alpha(z)=\varepsilon(z) \mu(z) .
$$

By performing the polynomial multiplication of both sides of (14) and by equating the coefficients of the same powers of $z$, it will be found that the equation associated with $z^{t}$ is precisely the generic equation under (12).

To derive the circulant representation of order $T$ of the ARMA equations, we impose the conditions that $y_{t}=y_{(t \bmod T)}$ and that $\varepsilon_{t}=\varepsilon_{(t \bmod T)}$. In terms of equation (11), the effect of these conditions is to replace the presample elements by elements from within the sample. Thus $y_{-1}=y_{T-1}, \ldots, y_{-p}=$ $y_{T-p}$ and, likewise, $\varepsilon_{-1}=\varepsilon_{T-1}, \ldots, \varepsilon_{-q}=\varepsilon_{T-q}$.

With these provisos, the polynomials of equation (14) can be converted to circulant matrices simply by replacing the complex argument $z$ by the matrix argument $K$.

\section{Spectral Factorisation of Circulant Matrices}

The matrix operator $K$ has a spectral factorisation which is particularly useful in analysing the properties of the discrete Fourier transform. To demonstrate this factorisation, we must first define the so-called Fourier matrix. This is a symmetric matrix $U=T^{-1 / 2}\left[W^{j t} ; t, j=0, \ldots, T-1\right]$ whose generic element in the $j$ th row and $t$ th column is

$$
\begin{gathered}
W^{j t}=\exp (-i 2 \pi t j / T)=\cos \left(\omega_{j} t\right)-\sin \left(\omega_{j} t\right), \\
\text { where } \omega_{j}=2 \pi j / T .
\end{gathered}
$$

On taking account of the $T$-periodicity of $W^{q}=\exp (-i 2 \pi q / T)$, the matrix can be written explicitly as

$$
U=\frac{1}{\sqrt{T}}\left[\begin{array}{ccccc}
1 & 1 & 1 & \ldots & 1 \\
1 & W & W^{2} & \ldots & W^{T-1} \\
1 & W^{2} & W^{4} & \ldots & W^{T-2} \\
\vdots & \vdots & \vdots & & \vdots \\
1 & W^{T-1} & W^{T-2} & \ldots & W
\end{array}\right] .
$$




\section{D.S.G. POLLOCK: Circulant Matrices}

The second row and the second column of this matrix contain the $T$ th roots of unity. The conjugate matrix is defined as $\bar{U}=T^{-1 / 2}\left[W^{-j t} ; t, j=0, \ldots, T-1\right]$; and, by using $W^{-q}=W^{T-q}$, this can be written explicitly as

$$
\bar{U}=\frac{1}{\sqrt{T}}\left[\begin{array}{ccccc}
1 & 1 & 1 & \ldots & 1 \\
1 & W^{T-1} & W^{T-2} & \ldots & W \\
1 & W^{T-2} & W^{T-4} & \ldots & W^{2} \\
\vdots & \vdots & \vdots & & \vdots \\
1 & W & W^{2} & \ldots & W^{T-1}
\end{array}\right] .
$$

It is readily confirmed that $U$ is a unitary matrix fulfilling the condition

$$
\bar{U} U=U \bar{U}=I .
$$

Now consider postmultiplying the unitary matrix $U$ by the diagonal matrix

$$
D=\left[\begin{array}{ccccc}
1 & 0 & 0 & \ldots & 0 \\
0 & W^{T-1} & 0 & \ldots & 0 \\
0 & 0 & W^{T-2} & \ldots & 0 \\
\vdots & \vdots & \vdots & \ddots & \vdots \\
0 & 0 & 0 & \ldots & W
\end{array}\right]
$$

Then it is easy to see that

$$
U D=K U,
$$

where $K$ is the circulant operator from (3). From this, it follows that $K=U D \bar{U}$ and, more generally, that

$$
K^{q}=U D^{q} \bar{U}=\bar{U} \bar{D}^{q} U
$$

where

$$
\bar{D}=\operatorname{diag}\left\{1, W, W^{2}, \ldots, W^{T-1}\right\}
$$

is both the conjugate and the inverse of $D$. The second equality of (21) follows from the fact that $K^{q}$ is a real-valued matrix which must equal its own conjugate. By similar means, it can be shown that

$$
K^{\prime}=K^{-1}=U \bar{D} \bar{U}=\bar{U} D U,
$$

The following conclusions can be reached in a straightforward manner:

$$
\text { If } A=\alpha(K) \text { is a circulant matrix then }
$$

$$
\begin{aligned}
\text { (i) } & A=\alpha(K)=U \alpha(D) \bar{U}=\bar{U} \alpha(\bar{D}) U, \\
\text { (ii) } & A^{\prime}=\alpha\left(K^{\prime}\right)=U \alpha(\bar{D}) \bar{U}=\bar{U} \alpha(D) U, \\
\text { (iii) } & A^{-1}=\alpha^{-1}(K)=U \alpha^{-1}(D) \bar{U} .
\end{aligned}
$$




\section{D.S.G. POLLOCK: Circulant Matrices}

We may describe the elements of the diagonal matrix $\alpha(\bar{D})$ as the spectral ordinates of the circulant matrix $A=\alpha(K)$. The $j$ th diagonal element of the matrix $\alpha(\bar{D})$ is given by

$$
\delta_{j}=\sum_{t} \alpha_{t} W^{j t}=\sum_{t}\left(\alpha_{t}^{r e}+i \alpha_{t}^{i m}\right)\left\{\cos \left(\omega_{j} t\right)-i \sin \left(\omega_{j} t\right)\right\}
$$

where $\omega_{j}=2 \pi j / T$.

In fact, the sequence $\left\{\delta_{0}, \delta_{1}, \ldots, \delta_{T-1}\right\}$ of the diagonal elements of $\alpha(\bar{D})$ is nothing but the discrete Fourier transform of the sequence $\left\{\alpha_{0}, \alpha_{1}, \ldots, \alpha_{T-1}\right\}$ of the elements in the leading vector of $A=\alpha(K)$. The reciprocal relationship of the two sequences is entailed in the following definition.

If $A=U \alpha(D) \bar{U}$ and $\Delta=U \delta(D) \bar{U}$ are circulant matrices such that $\Delta=U A$ and $A=\bar{U} \Delta$, then they are described as Fourier reciprocals. The conditions relating the matrices imply that $\alpha(\bar{D})=$ $\bar{U} \delta(\bar{D})$ and $\delta(D)=U \alpha(D)$.

The restrictions of realness and symmetry, which may be imposed on the matrix $A=\alpha(K)$, have direct implications for the elements of the matrix $\alpha(D)$. If $A=\alpha(K)$ is a real-valued matrix, which is to say that $\alpha_{t}^{i m}=0$ for all $t$, then the sequence of complex numbers $\left\{\delta_{0}, \delta_{1}, \ldots, \delta_{T-1}\right\}$, defined according to (25), which are the diagonal elements of $\alpha(\bar{D})$, consists of a real part which is an even or symmetric function of the index $t$ and an imaginary part which is an odd or anti-symmetric function.

If $A=A^{\prime}$ is a real-valued symmetric circulant matrix, such that $\alpha_{j}=\alpha_{T-j}$ then $\alpha(D)=\alpha(\bar{D})$, which is to say that the diagonal elements of $\alpha(\bar{D})$ constitute a real even sequence such that $\delta_{j}=$ $\delta_{T-j}$.

Of course, the condition of matrix symmetry, which is that $\alpha_{\tau}=\alpha_{T-\tau}$, ensures that the terms in the sine function cancel within the sum defining $\delta_{j}$ in (25). Thus, when $A$ is a real symmetric circulant matrix, its spectral ordinates $\delta_{j}=$ $\sum_{t} \alpha_{t} \cos \left(\omega_{j} t\right)$ are the products of a cosine Fourier transform which therefore constitute a real-valued even sequence.

Since the sequences $\left\{\alpha_{0}, \alpha_{1}, \ldots, \alpha_{T-1}\right\}$ and $\left\{\delta_{0}, \delta_{1}, \ldots, \delta_{T-1}\right\}$ bear a reciprocal relationship to each other, it follows that, if one of them is realvalued and even, then so must be the other. Thus, according to (26), there are $\alpha(\bar{D})=\bar{U} \delta(\bar{D})$ and $\alpha(D)=\bar{U} \delta(D)$. But, if $\alpha(\bar{D})=\alpha(D)$, as in (27), then $\delta(\bar{D})=\delta(D)$. Therefore, the converse of $(27)$ is true:

If the diagonal elements of $\alpha(\bar{D})$ constitute a sequence which is real and even such that $\delta_{j}=\delta_{T-j}$, then $A=\bar{U} \alpha(\bar{D}) U$ is a real symmetric circulant matrix, such that $\alpha_{j}=\alpha_{T-j}$. 


\section{D.S.G. POLLOCK: Circulant Matrices}

Example. Consider an ARMA process represented, in the form of an infiniteorder moving-average, by $y(t)=\phi(L) \varepsilon(t)$, where $\phi(L)=\mu(L) / \alpha(L)$ is a ratio of polynomials in the lag operator. The autocovariance generating function of the process is

$$
\gamma(z)=\sigma^{2} \phi(z) \phi\left(z^{-1}\right)=\sum_{\tau=0}^{\infty} \gamma_{\tau}\left(z^{\tau}+z^{-\tau}\right),
$$

where $\gamma_{\tau}=\sigma^{2} \sum_{j=0}^{\infty} \phi_{j} \phi_{j+\tau}$ is the autocovariance of lag $\tau$.

Setting $z=\exp \left\{i \omega_{j}\right\}$ and recognising that $\exp \left\{i \omega_{j} \tau\right\}+\exp \left\{-i \omega_{j} \tau\right\}=$ $2 \cos \left(\omega_{j} \tau\right)$ gives an ordinate of the spectral density function in the form of

$$
f\left(\omega_{j}\right)=\frac{1}{2 \pi} \gamma\left(e^{i \omega_{j}}\right)=\frac{1}{2 \pi}\left\{\gamma_{0}+2 \sum_{\tau=1}^{\infty} \gamma_{\tau} \cos \left(\omega_{j} \tau\right)\right\}
$$

which represents the cosine Fourier transform of the sequence of ordinary autocovariances. The spectral ordinates, which are indexed by $j=0, \ldots, T-1$, constitute an even real-valued sequence.

The same spectral ordinates can be obtained from the elements of the diagonal matrix

$$
\gamma(D)=\bar{U} \gamma(K) U=\bar{U} \phi\left(K^{\prime}\right) \phi(K) U
$$

where $\gamma(K)=\Gamma$ and $\phi(K)=\Phi$ are the circulant matrices which are obtained when $z$ is replaced by $K$ in $\gamma(z)$ and $\phi(z)$ respectively.

Observe that, whereas, in general, the coefficients of $\gamma(z)$ constitute an infinite sequence, the coefficients of $\gamma(D)$ form only a finite sequence (or a periodic sequence) of order $T$ which is real and even. The relationship between the coefficients of $\gamma(z)$ and those of $\gamma(D)=\psi(D)$ is expressed in equation (6).

\section{The Fourier Transform of a Stationary Process}

Let $y=\left[y_{0}, y_{1}, \ldots, y_{T-1}\right]^{\prime}$ be a vector of observations on a stationary stochastic process of zero mean, and consider the equation

$$
Y=y(K)=U y(D) \bar{U}=\bar{U} y(\bar{D}) U
$$

which defines a real-valued circulant matrix $Y$ equal to its own conjugate. Observe that, if $e_{0}=[1,0, \ldots, 0]^{\prime}$ is the leading vector of the identity matrix of order $T$, then

$$
\begin{aligned}
Y e_{0} & =y=\left[y_{0}, y_{1}, \ldots, y_{T-1}\right]^{\prime}, \\
T^{1 / 2} U e_{0} & =T^{1 / 2} \bar{U} e_{0}=i=[1,1, \ldots, 1]^{\prime}, \\
y(D) i & =T \zeta^{*}=T\left[\zeta_{T-1}, \zeta_{T-2}, \ldots, \zeta_{0}\right]^{\prime} \text { and } \\
y(\bar{D}) i & =T \zeta=T\left[\zeta_{0}, \zeta_{1}, \ldots, \zeta_{T-1}\right]^{\prime} .
\end{aligned}
$$




\section{D.S.G. POLLOCK: Circulant Matrices}

Here, $\zeta$ is the statistical form of the discrete Fourier transform (DFT) of the vector $y$. Its elements are the values $\left\{\zeta_{k}=T^{-1} y\left(z_{k}\right) ; k=0, \ldots, T-1\right\}$ which come from setting $z=z_{k}=\exp (-2 \pi k / T)$ in $y(z)$ which is the $z$-transform of the data sequence. It differs from the DFT defined in the previous section by a scalar factor, which is $T^{-1}$.

Premultiplying the equation $Y=\bar{U} y(\bar{D}) U$ by $U$ and postmultiplying it by $e_{0}$ gives

$$
U y=T^{1 / 2} \zeta
$$

which represents the direct DFT of the vector $y$. Premultiplying the latter equation by $\bar{U}$ gives

$$
\bar{U} \zeta=T^{-1 / 2} y
$$

and this represents the inverse transform by which $y$ is recovered from $\zeta$.

Since $y$ is a real-valued vector, its complex-valued transform $\zeta$ is subject to the condition that its real part forms an even or symmetric sequence whilst its imaginary part forms an odd or antisymmetric sequence. Thus, if $\zeta_{j}=$ $\zeta^{r e}+i \zeta^{i m}$, then $\zeta_{T-j}=\zeta^{r e}-i \zeta^{i m}=\zeta_{j}^{*}$ is the complex conjugate of $\zeta_{j}$.

Example. To reveal the nature of these transforms in more detail, we may examine the generic elements of the expressions. In the case of equation (34), which represents the direct DFT, there is

$$
\begin{aligned}
\zeta_{j} & =\frac{1}{T} \sum_{t=0}^{T-1} y_{t} e^{-i \omega_{j} t} \\
& =\frac{1}{T} \sum_{t=0}^{T-1}\left\{y_{t} \cos \left(\omega_{j} t\right)-i y_{t} \sin \left(\omega_{j} t\right)\right\},
\end{aligned}
$$

where we have used the identity $\exp \left(-i \omega_{j} t\right)=\cos \left(\omega_{j} t\right)-i \sin \left(\omega_{j} t\right)$. Observe that, with $\omega_{j}=2 \pi j / T$, there are $\cos \left(\omega_{0} t\right)=1$ and $\sin \left(\omega_{0} t\right)=0$. Also, if $T=2 n$ is even, then $\omega_{n}=\pi$, so that $\cos \left(\omega_{n} t\right)=(-1)^{t}$ and $\sin \left(\omega_{n} t\right)=0$. Therefore, equation (36) is amenable to various specialisations. First, there is

$$
\zeta_{0}=\alpha_{0}=\frac{1}{T} \sum_{t=0}^{T-1} y_{t}=\bar{y} .
$$

Next, there are

$$
\zeta_{j}=\frac{\alpha_{j}-i \beta_{j}}{2} \quad \text { and } \quad \zeta_{T-j}=\zeta_{j}^{*}=\frac{\alpha_{j}+i \beta_{j}}{2},
$$

with

$$
\alpha_{j}=\frac{2}{T} \sum_{t=0}^{T-1} y_{t} \cos \left(\omega_{j} t\right) \quad \text { and } \quad \beta_{j}=\frac{2}{T} \sum_{t=0}^{T-1} y_{t}\left(\sin \omega_{j} t\right)
$$


where $j=1, \ldots, n=(T-1) / 2$ if $T=2 n+1$ is odd and $j=1, \ldots, n-1=$ $(T / 2)-1$ if $T=2 n$ is even. Finally, there is

$$
\zeta_{n}=\alpha_{n}=\frac{1}{T} \sum_{t=0}^{T-1}(-1)^{t} y_{t} \quad \text { if } T=2 n \text { is even. }
$$

Observe that we have, in effect, defined the integer

$$
n=[T / 2]= \begin{cases}T / 2, & \text { if } T \text { is even } \\ (T-1) / 2, & \text { if } T \text { is odd }\end{cases}
$$

Here, $[T / 2]=\operatorname{trunc}(T / 2)$ represents the integer truncation of the rational number $T / 2$ which comes from discarding its decimal part, if any. There is also

$$
[(T-1) / 2]= \begin{cases}n=(T-1) / 2, & \text { if } T \text { is odd } \\ n-1=(T / 2)-1, & \text { if } T \text { is even }\end{cases}
$$

In the case of equation (35), which represents the inverse DFT, there is

$$
\begin{aligned}
y_{t} & =\sum_{j=0}^{T-1} \zeta_{j} e^{i \omega_{j} t} \\
& =\sum_{j=0}^{T-1}\left\{\zeta_{j} \cos \left(\omega_{j} t\right)+i \zeta_{j} \sin \left(\omega_{j} t\right)\right\} .
\end{aligned}
$$

Equation (38) implies that

$$
\alpha_{j}=\zeta_{j}+\zeta_{j}^{*} \quad \text { and } \quad \beta_{j}=-i\left(\zeta_{j}-\zeta_{j}^{*}\right)
$$

where $j=1, \ldots, n$ if $T$ is odd and $j=1, \ldots, n-1$ if $T$ is even, which is to say that $j=1, \ldots,[(T-1) / 2]$. Therefore, on the understanding that $\beta_{0}=0$ and that $\beta_{n}=0$ if $T$ is odd, equation (43) can be written as

$$
\begin{aligned}
y_{t} & =\sum_{j=0}^{n}\left\{\alpha_{j} \cos \left(\omega_{j} t\right)+\beta_{j} \sin \left(\omega_{j} t\right)\right\} \\
& =\sum_{j=0}^{n} \rho_{j} \cos \left(\omega_{j} t-\theta_{j}\right),
\end{aligned}
$$

where

$$
\rho_{j}^{2}=\alpha_{j}^{2}+\beta_{j}^{2} \quad \text { and } \quad \theta_{j}=\tan ^{-1}\left(\beta_{j} / \alpha_{j}\right)
$$




\section{D.S.G. POLLOCK: Circulant Matrices}

Equation (45) indicates that the data sequence can be expressed uniquely in terms of a sum of cosine functions of rising frequency, each with a particular amplitude $\rho_{j}$ and a particular phase displacement $\theta_{j}$. The sequence of the squared amplitudes, weighted by the variances of the accompanying trigonometrical functions, constitutes the periodogram of the data, whilst the sequence of phase angles constitutes its phase spectrum.

Whereas our intuition might be served better by expressing the Fourier transform in terms of ordinary trigonometrical functions, it greatly eases the burden of notation if we use complex exponentials instead. The use of matrix notation results in a further economy of expression. Nevertheless, one should be aware of the basis of the complex expressions. In particular, the unitary nature of the Fourier matrix stems for the orthogonality of the sequences of sine and cosine ordinates.

Example. The vectors $c_{i}=\left[\cos \left(\omega_{i} t\right) ; t=0, \ldots, n\right]^{\prime}$ and $s_{j}=\left[\sin \left(\omega_{j} t\right) ; t=\right.$ $0, \ldots, n]^{\prime}$ are subject to some conditions of orthogonality provided that $\omega_{i}$ and $\omega_{j}$ belong to the set of Fourier frequencies $\left\{\omega_{j}=2 \pi j / T ; j=1, \ldots, n\right\}$. In that case, the following conditions prevail:

$$
\begin{array}{ll}
c_{i}^{\prime} c_{j}=0 & \text { if } \quad i \neq j, \\
s_{i}^{\prime} s_{j}=0 & \text { if } \quad i \neq j, \\
c_{i}^{\prime} s_{j}=0 & \text { for all } i, j .
\end{array}
$$

In addition, there are some sums of squares which can be taken into account in computing the coefficients of the Fourier decomposition:

$$
\left.\begin{array}{rl}
c_{0}^{\prime} c_{0} & =T, \\
s_{0}^{\prime} s_{0} & =0, \\
c_{j}^{\prime} c_{j} & =\frac{1}{2} T, \\
s_{j}^{\prime} s_{j} & =\frac{1}{2} T .
\end{array}\right\} \quad \text { for } \quad j=1, \ldots, n-1 .
$$

For $j=n$, there are

$$
\begin{aligned}
& \left.\begin{array}{l}
s_{n}^{\prime} s_{n}=\frac{1}{2} T, \\
c_{n}^{\prime} c_{n}=\frac{1}{2} T,
\end{array}\right\} \quad \text { if } \quad 2 n=T-1, \\
& \left.\begin{array}{l}
s_{n}^{\prime} s_{n}=0, \\
c_{n}^{\prime} c_{n}=T .
\end{array}\right\} \quad \text { if } \quad 2 n=T ;
\end{aligned}
$$

which correspond, respectively, to the cases where $T$ is odd and $T$ is even.

The latter relationships enable us to rewrite the expressions for $\alpha_{j}$ and $\beta_{j}$, where $j=1, \ldots,[(T-1) / 2]$, which are found under $(39)$, as

$$
\alpha_{j}=\frac{2}{T} c_{j}^{\prime} y=\left(c_{j}^{\prime} c_{j}\right)^{-1} c_{j}^{\prime} y \quad \text { and } \quad \beta_{j}=\frac{2}{T} s_{j}^{\prime} y=\left(s_{j}^{\prime} s_{j}\right)^{-1} s_{j}^{\prime} y .
$$




\section{D.S.G. POLLOCK: Circulant Matrices}

The orthogonality conditions indicate that $\alpha_{i}$ and $\alpha_{j}$ are uncorrelated when $i \neq j$ as are $\beta_{i}$ and $\beta_{j}$. Moreover, $\alpha_{i}$ and $\beta_{j}$ are uncorrelated for all $i, j$. Thus

$$
\begin{gathered}
C\left(\alpha_{i}, \alpha_{j}\right)=C\left(\beta_{i}, \beta_{j}\right)=0 \text { when } i \neq j \\
\text { and } C\left(\alpha_{i}, \beta_{j}\right)=0 \text { for all } i, j .
\end{gathered}
$$

\section{The Periodogram and the Circular Autocovariances}

The periodogram can be expressed in terms of the circulant matrix $G=$ $T^{-1} Y^{\prime} Y$ which contains the so-called circular autocovariances of the data. These values $g_{0}, g_{1}, \ldots, g_{T-1}$ are given by the formula

$$
g_{\tau}=\frac{1}{T} \sum_{t=0}^{T-1} y_{t} y_{t+\tau} ; \quad \text { where } \quad y_{t}=y_{(t \bmod T)}
$$

$$
\text { or, equivalently, }
$$

$$
g_{\tau}=\frac{1}{T} \sum_{t=0}^{T-1-\tau} y_{t} y_{t+\tau}+\frac{1}{T} \sum_{t=0}^{\tau-1} y_{t} y_{t+T-\tau} .
$$

They differ from the ordinary autocovariances which are obtained from the first formula by setting $y_{t}=0$ when $t>T-1$ or from the second formula by taking only the leading sum.

The matrix of circular autocovariances can be expressed variously as

$$
\begin{aligned}
G & =\bar{U} g(D) U \\
& =T^{-1} Y^{\prime} Y=T^{-1} \bar{U} y(\bar{D}) y(D) U .
\end{aligned}
$$

The core of this expression is the real-valued diagonal matrix

$$
g(D)=T^{-1} y(\bar{D}) y(D)=T \operatorname{Diag}\left\{\left|\zeta_{0}\right|^{2},\left|\zeta_{1}\right|^{2}, \ldots,\left|\zeta_{T-1}\right|^{2}\right\}
$$

Consider premultiplying equation (53) by $e_{0}^{\prime}$ and postmultiplying it by $e_{0}$. Since $e_{0}^{\prime} G e_{0}=g_{0}$ and $U e_{0}=\bar{U} e_{0}=T^{-1 / 2} i$, we get the following expression for the variance:

$$
g_{0}=T^{-2} i^{\prime} y(\bar{D}) y(D) i=\sum_{j=0}^{T-1}\left|\zeta_{j}\right|^{2} .
$$

The terms in the sum on RHS can be specialised in various ways. When $j=0$, there is

$$
\left|\zeta_{0}\right|^{2}=\alpha_{0}^{2}=\bar{y}^{2}
$$


When $j=1, \ldots,[(T-1) / 2]$, there are

$$
\left|\zeta_{j}\right|^{2}=\left|\zeta_{T-j}\right|^{2}=\frac{\alpha_{j}^{2}+\beta_{j}^{2}}{4}=\frac{\rho_{j}^{2}}{4}
$$

Finally, there is

$$
\left|\zeta_{n}\right|^{2}=\alpha_{n}^{2} \quad \text { if } \quad T=2 n \quad \text { is even. }
$$

The terms $\left|\zeta_{j}\right|^{2}$ and $\left|\zeta_{T-j}\right|^{2}$ can be combined within the sum of equation (55). It follows that, when $T$ is even, the equation can be rewritten as

$$
g_{0}=\alpha_{0}^{2}+\sum_{j=0}^{n-1} \frac{\rho_{j}^{2}}{2}+\alpha_{n}^{2} .
$$

When $T$ is odd, the summation is extended to include the $n$th term which becomes $\rho_{n}^{2} / 2=\left(\alpha_{n}^{2}+\beta_{n}^{2}\right) / 2$. Observe that $g_{0}-\alpha_{0}^{2}=T^{-1} \sum_{t}\left(y_{t}-\bar{y}\right)^{2}$ is the variance calculated from the mean-adjusted data.

The sequence $\left\{T \rho_{1}^{2} / 2, T \rho_{1}^{2} / 2, \ldots, T \rho_{n-1}^{2} / 2, T \alpha_{n}^{2}\right\}$, which applies when $T$ is even, together with the alternative sequence $\left\{T \rho_{1}^{2} / 2, T \rho_{1}^{2} / 2, \ldots, T \rho_{n}^{2} / 2\right\}$, which applies when $T$ is odd, constitute the periodogram of the data. Equation (59) represents an analysis of variance in terms of the periodogram. to give

Consider premultiplying equation (53) by $U$ and postmultiplying it by $e_{0}$

$$
\begin{aligned}
U G e_{0} & =g(D) U e_{0} \\
& =T^{-1} y(\bar{D}) y(D) U e_{0} .
\end{aligned}
$$

From (33), we have the identity $T^{1 / 2} U e_{0}=i$. Therefore, $T^{1 / 2} g(D) U e_{0}=$ $g(D) i=\nu$ is the vector of the rescaled periodogram ordinates. Moreover $G e_{0}=$ $g$ is the vector of circular autocovariances. It follows that

$$
T^{1 / 2} \nu=U g \quad \text { and } \quad g=T^{1 / 2} \bar{U} \nu
$$

which is to say that the vector $\nu=\left[\left|\zeta_{0}\right|^{2},\left|\zeta_{1}\right|^{2}, \ldots,\left|\zeta_{T-1}\right|^{2}\right]^{\prime}$ of rescaled periodogram ordinates and the vector $g$ of circular autocovariances are related to each other via a discrete Fourier transform.

It is clear, from equation (60), that there are two strategies that can be pursued in calculating the ordinates of the periodogram. The first strategy entails finding the vector $g$ of autocovariances and then subjecting it to a Fourier transform. The second strategy is to find vector $y(\bar{D}) i=T^{1 / 2} \zeta$ by applying the Fourier transform of equation (34) to the data vector $y$ and then to use the elements of $U y=T^{1 / 2} \zeta$ in finding the ordinates of the periodogram according to equations $(56)-(58)$. 


\section{D.S.G. POLLOCK: Circulant Matrices}

Example. In equation (53), the matrix $U$ represents a set of complex eigenvectors of the matrix $G$. The diagonal matrix $g(D)$ contains the corresponding set of real-valued eigenvalues. A set of real-valued eigenvectors can be found to replace the complex ones. Given that $G$ is a real-valued matrix, it follow from (53) that

$$
G U=U g(D) \quad \text { and } \quad G \bar{U}=\bar{U} g(D)
$$

By combining these equations in two ways, we can write

$$
G C=g(D) C \quad \text { and } \quad G S=g(D) S,
$$

where

$$
C=\left(\frac{U+\bar{U}}{2}\right) \quad \text { and } \quad S=\left(\frac{U-\bar{U}}{2 i}\right)
$$

are, respectively, a matrix of cosines and a matrix of sines.

To reveal the structure of these matrices, we may use the notation of the example following equation (46) whereby $c_{i}=\left[\cos \left(\omega_{i} t\right) ; t=0, \ldots, n\right]^{\prime}$ and $s_{j}=$ $\left[\sin \left(\omega_{j} t\right) ; t=0, \ldots, n\right]^{\prime}$. Also, let $i=[1,1, \ldots, 1]^{\prime}$ and $j=\left[1,-1, \ldots,(-1)^{T-1}\right]^{\prime}$. Then

$$
C= \begin{cases}{\left[i, c_{1}, \ldots, c_{n-1}, j, c_{n-1}, \ldots, c_{1}\right],} & \text { if } T \text { is even } \\ {\left[i, c_{1}, \ldots, c_{n-1}, c_{n}, c_{n}, \ldots, c_{1}\right],} & \text { if } T \text { is odd }\end{cases}
$$

Likewise,

$$
S= \begin{cases}{\left[0, s_{1}, \ldots, s_{n-1}, 0,-s_{n-1}, \ldots,-s_{1}\right],} & \text { if } T \text { is even } \\ {\left[0, s_{1}, \ldots, s_{n-1}, s_{n},-s_{n}, \ldots,-s_{1}\right],} & \text { if } T \text { is odd }\end{cases}
$$

Now define the matrix which comprises the distinct elements of $C$ and $S$ ordered by rising frequency. This is

$$
V= \begin{cases}{\left[i, c_{1}, s_{1}, \ldots, c_{n-1}, s_{n-1}, j\right],} & \text { if } T \text { is even } \\ {\left[i, c_{1}, s_{1}, \ldots, s_{n-1}, c_{n}, s_{n}\right],} & \text { if } T \text { is odd }\end{cases}
$$

The matrix $V$ is square and of full rank, and it satisfies the equation

$$
G V=g(D) V
$$

Therefore, we can describe the vectors of sines and cosines within $V$ as the characteristic vectors of the matrix of circular autocovariances.

When the characteristic vectors are ordered according to the decreasing sizes of their associated eigenvalues, they constitute the so-called principal 


\section{D.S.G. POLLOCK: Circulant Matrices}

components of the matrix $Y$. The concept of principal components supplies additional meaning to the analysis of the periodogram.

An analysis of the principal components of the data has been the basis of a number of recent papers in climatology which purport to reveal the presence in meteorological phenomena of hidden cycles. (See, for example, Schlesinger and Ramankutty [12] and Vautard and Ghil [13].)

The procedure of uncovering the cycles, which is known as singular spectral analysis, is not based upon circulant matrices. Instead, it is based, typically, upon a data matrix which can be obtained from the matrix of equation (11) by replacing the presample elements by zeros and by appending an upper triangular matrix at the bottom whose nonzero elements are the remainder of the sample values.

The reality of the climatological cycles has been disputed; and those who claim to have uncovered them seem to be unable to provide convincing proof. If the analysis were conducted using circulant matrices, then it should be straightforward to employ standard techniques to assess whether the periodogram ordinates are significantly different from those which might be generated from a white-noise process.

\section{Complex Normal Distributions}

In this section, we shall develop the statistical properties of a complex normal vector which is obtained by applying a Fourier transform to a spherical normal vector $y \sim N\left(0, \sigma^{2} I\right)$, with a zero mean vector $E(y)=0$ and with dispersion matrix $D(y)=\sigma^{2} I$, which represents a segment of a white-noise process. The results will be used in the following section in analysing the statistical properties of the periodogram of a normal vector $y \sim N\left(0, \sigma^{2} Q\right)$ obtained by sampling an arbitrary stationary stochastic process.

The basic results, which are exact, are obtained under the assumption that $y$ is from a circular process and that, accordingly, $Q$ is a circulant matrix. These results provide asymptotically valid approximations for the more general and realistic cases where $y$ is generated by a linear stochastic process which is not necessarily a normal process.

In order to develop the statistical theory of a complex normal vector, we need to generalise some of the familiar operations of multivariate statistical analysis.

First we define the expectation of a complex random vector:

If $\zeta$ is a complex vector with a generic element of $\zeta_{j}=\gamma_{j}+i \delta_{j}$, then $E(\zeta)$ is a vector whose generic element is $E\left(\gamma_{j}\right)+i E\left(\delta_{j}\right)$.

Next we define the dispersion matrix of a complex vector:

If $\zeta$ is a complex vector of zero mean with a generic element of $\zeta_{j}=\gamma_{j}+i \delta_{j}$, then its variance-covariance matrix or dispersion matrix $D(\zeta)=E\left(\zeta \bar{\zeta}^{\prime}\right)=Q$ has $E\left(\zeta_{i} \zeta_{j}^{*}\right)=E\left\{\left(\gamma_{i}+i \delta_{i}\right)\left(\gamma_{j}-i \delta_{j}\right)\right\}$ as the element in the $i, j$ th position. 


\section{D.S.G. POLLOCK: Circulant Matrices}

In general, the dispersion matrix of a complex vector is a Hermitian matrix $Q=\bar{Q}^{\prime}$ which is equal to its own conjugate transpose. However, if the real and imaginary parts of the complex vector $\zeta=\gamma+i \delta$ are uncorrelated, then its dispersion matrix $Q=Q^{\prime}$ is real and symmetric. Of particular interest is the dispersion matrix of a complex vector $\zeta=T^{-1 / 2} U y$ which represents the Fourier transform of a real-valued normal vector $y$. In that case, since the elements of $\gamma$ and $\delta$ are mutually uncorrelated, the dispersion matrix is real and symmetric.

We are interested, particularly, in the statistical properties of the Fourier transform of a real-valued normal vector. The simplest case is that of a vector of independently and identically distributed elements:

If $y \sim N\left(0, \sigma^{2} I_{T}\right)$ is a real-valued vector with a spherical normal distribution and if $U$ is a unitary transformation, then $T^{1 / 2} \zeta=$ $U y \sim N_{C}\left(0, \sigma^{2} I_{T}\right)$ has a complex spherical normal distribution.

Here the subscripted $C$ on $N_{C}$ indicates that this is the distribution of a complex vector.

Notice that the elements of $\zeta$ are not statistically independent in the ordinary sense since, if it is the transform of a real-valued vector $y$, then $\zeta$ will comprise both $\zeta_{j}=\gamma_{j}+i \delta_{j}$ and its conjugate $\zeta_{T-j}=\gamma_{j}-i \delta_{j}$. However the complex coordinates $\gamma_{j}, \delta_{j} ; j=0, \ldots, n=[T / 2]$ will be mutually uncorrelated, as will be the corresponding complex moduli $\rho_{j}^{2}=\gamma_{j}^{2}+\delta_{j}^{2}$.

It is straightforward to associate a chi-square distribution with the modulus of a complex normal vector with a spherical distribution.

Let $T^{1 / 2} \zeta=U y \sim N_{C}\left(0, \sigma^{2} I_{T}\right)$ be a complex spherical normal vector which represents the Fourier transform of a real-valued spherical normal vector $y \sim N\left(0, \sigma^{2} I_{T}\right)$. Then $T \bar{\zeta}^{\prime} \zeta / \sigma^{2}=$ $y^{\prime} y / \sigma^{2} \sim \chi^{2}(T)$.

According to Cochrane's theorem, if $y \sim N\left(0, \sigma^{2} I_{T}\right)$ and if $P=P^{\prime}=P^{2}$ is a real-valued symmetric matrix of order $T$ with $\operatorname{rank}(P)=r$, then $y^{\prime} P y / \sigma^{2} \sim$ $\chi^{2}(r)$ and $y^{\prime}(I-P) y / \sigma^{2} \sim \chi^{2}(T-r)$ are statistically independent chi-square variates. The following is a specialisation of the result which is appropriate to complex spherical normal vectors:

Let $T^{1 / 2} \zeta=U y \sim N_{C}\left(0, \sigma^{2} I_{T}\right)$ be a complex spherical normal vector obtained from the real-valued vector $y$ via the unitary Fourier matrix $U$ of (16). Let $S=S^{\prime}=S^{2}$ be a realvalued selection matrix of order $T$ with $r$ units and $T-r$ zeros on the diagonal, which form an even sequence, and with zeros elsewhere. Then $\bar{U} S U=P=P^{\prime}=P^{2}$ is a real-valued symmetric idempotent circulant matrix with $\operatorname{rank}(P)=r$; and it follows that $T \bar{\zeta} S \zeta / \sigma^{2}=y^{\prime} \bar{U} S U y / \sigma^{2} \sim \chi^{2}(r)$ and $T \bar{\zeta}^{\prime}(I-S) \zeta / \sigma^{2}=$ $y^{\prime} \bar{U}(I-S) U y / \sigma^{2} \sim \chi^{2}(T-r)$ are statistically independent.

Here, we should recall that, according to (28), the condition that the diagonal elements of $S$ constitute an even sequence is sufficient to ensure that 


\section{D.S.G. POLLOCK: Circulant Matrices}

$P=\bar{U} S U$ is a real-valued matrix. We should also recall that, in general, a realvalued symmetric idempotent matrix can be expressed $P=Q D Q^{\prime}$, where $Q$, which is a normalised version of the matrix $V$ of (67), is a real-valued orthonormal matrix such that $Q Q^{\prime}=Q^{\prime} Q=I$, and where $D$ is a matrix with units and zeros on the diagonal and with zeros elsewhere. Therefore, it is possible to circumvent the complex notation.

Example. Let $\zeta=T^{-1 / 2} U y$ be the Fourier transform of a real-valued vector $y \sim N\left(0, \sigma^{2} I_{T}\right)$. Let $\zeta_{j}=\left(\alpha_{j}+i \beta_{j}\right) / 2$ and let $S_{j}=e_{j} e_{j}^{\prime}+e_{T-j} e_{T-j}^{\prime}$, where $0<j \leq[(T-1) / 2]$. Then $S_{j}$ is a diagonal matrix of rank 2 with units on the diagonal in the positions $j$ and $T-j$ and with zeros elsewhere. The diagonal elements of $S_{j}$ constitute a real and even sequence. Therefore, it follows from (28) that $\bar{U} S_{j} U$ is a real-valued symmetric idempotent circulant matrix. Hence

$$
\begin{aligned}
\frac{T}{\sigma^{2}} \bar{\zeta}^{\prime} S_{j} \zeta & =\frac{T}{\sigma^{2}}\left(\zeta_{j} \zeta_{j}^{*}+\zeta_{T-j} \zeta_{T-j}^{*}\right) \\
& =\frac{T}{2 \sigma^{2}}\left(\alpha_{j}^{2}+\beta_{j}^{2}\right)=\frac{T}{2 \sigma^{2}} \rho_{j}^{2} \sim \chi(2) .
\end{aligned}
$$

If $T=2 n$ is even, then both $S_{0}=e_{0} e_{0}^{\prime}$ and $S_{n}=e_{n} e_{n}^{\prime}$ are symmetric idempotent matrices of rank 1 whose diagonal elements constitute even sequences. In that case, both $\bar{U} S_{0} U$ and $\bar{U} S_{n} U$ are real-valued symmetric idempotent circulant matrices of rank 1 and, therefore,

$$
\begin{aligned}
& \frac{T}{\sigma^{2}} \zeta_{0} \zeta_{0}^{*}=T \frac{\alpha_{0}^{2}}{\sigma^{2}}=T \frac{\bar{y}^{2}}{\sigma^{2}} \sim \chi^{2}(1) \\
& \text { and } \frac{T}{\sigma^{2}} \zeta_{n} \zeta_{n}^{*}=T \frac{\alpha_{n}^{2}}{\sigma^{2}} \sim \chi^{2}(1) .
\end{aligned}
$$

If $T=2 n+1$ is odd, then $\beta_{n} \neq 0$ and there are $\zeta_{n}=\left(\alpha_{n}+i \beta_{n}\right) / 2$ and $\zeta_{T-n}=\left(\alpha_{n}-i \beta_{n}\right) / 2$, which are adjacent values within the vector $\zeta$. These give rise to a $\chi^{2}(2)$ variate in the manner of $(74)$.

\section{Statistical Properties of the Periodogram}

To see the significance for the analysis of the periodogram of the results of the foregoing section, let us consider the ARMA process of (10), which can be written in the form of $y(t)=\phi(L) \varepsilon(t)$, where $\phi(L)=\mu(L) / \alpha(L)$ represents the series expansion of the rational operator. The process can also be represented, in terms of the $z$-transform polynomials of (13), by putting $y(z)=\phi(z) \varepsilon(z)$.

The analysis proceeds by using the circulant version of the ARMA process. By putting $\varepsilon_{t} \bmod T$ in place of $\varepsilon_{t}$, which replaces the elements outside the sample by elements from within the sample, and by replacing the complex argument $z$ by the matrix argument $K$, we derive the matrix equation $Y=\Phi \mathcal{E}$, where $Y=y(K), \Phi=\phi(K)$ and $\mathcal{E}=\varepsilon(K)$. The equation of the circulant process, together with that of its Fourier transform, can therefore be expressed as

$$
y=\Phi \varepsilon=\mathcal{E} \phi \quad \text { and } \quad T^{-1 / 2} U y=\zeta_{y}=\phi(D) \zeta_{\varepsilon},
$$


where $y=Y e_{0}, \phi=\Phi e_{0}$ and $\varepsilon=\mathcal{E} e_{0}$ are the leading vectors of their respective matrices.

We shall assume that $y \sim N\left(0, \sigma^{2} Q\right)$, where $Q=\Phi^{\prime} \Phi$, which implies that $\varepsilon=\Phi^{-1} y \sim N\left(0, \sigma^{2} I\right)$. The results of the previous section can be applied directly to the Fourier transform of $\varepsilon$ which is $\zeta_{\varepsilon}=\phi^{-1}(D) \zeta_{y}$.

Let $S_{k}=e_{k} e_{k}^{\prime}$ be the selection matrix with a unit in the $k$ th diagonal position and with zeros elsewhere. Then

$$
\begin{aligned}
\zeta_{\varepsilon k}^{\prime} \zeta_{\varepsilon k} & =\bar{\zeta}_{\varepsilon} S_{k} \zeta_{\varepsilon}^{*} \\
& =\bar{\zeta}_{y}^{\prime} \phi^{-1}(\bar{D}) S_{k} \phi^{-1}(D) \zeta_{y} \\
& =\frac{\zeta_{y k} \zeta_{y k}^{*}}{q_{k}},
\end{aligned}
$$

where $q_{k}$ is the $k$ th diagonal element of the matrix $\bar{U} Q U=q(D)=\phi(\bar{D}) \phi(D)$. Therefore, by putting $\zeta_{y k} \zeta_{y k}^{*} / q_{j}$ in place of $\zeta_{j} \zeta_{j}^{*}$ in equation (74), we get

$$
\frac{T}{\sigma^{2} q_{j}}\left\{\zeta_{y j} \zeta_{y j}^{*}+\zeta_{y(T-j)} \zeta_{y(T-j)}^{*}\right\}=\frac{T}{2 \sigma^{2} q_{j}} \rho_{y j}^{2} \sim \chi(2)
$$

where $j=1, \ldots,[(T-1) / 2]$. If $T=2 n$ is even, then, in addition,

$$
\begin{aligned}
& \frac{T}{\sigma^{2} q_{0}} \zeta_{0} \zeta_{0}^{*}=T \frac{\alpha_{0}^{2}}{\sigma^{2} q_{0}}=T \frac{\bar{y}^{2}}{\sigma^{2} q_{0}} \sim \chi^{2}(1) \\
& \text { and } \frac{T}{\sigma^{2} q_{n}} \zeta_{n} \zeta_{n}^{*}=T \frac{\alpha_{n}^{2}}{\sigma^{2} q_{n}} \sim \chi^{2}(1) .
\end{aligned}
$$

If $T=2 n+1$ is odd, then $\zeta_{n}$ and $\zeta_{T-n}$ are adjacent values within the vector $\zeta$. These give rise to a $\chi^{2}(2)$ variate in the manner of (78).

Now recall that the expected value of a $\chi^{2}(r)$ variate is $r$ whilst its variance is $2 r$. Also recall that, according to (30), the value of the spectral density function at the frequency $\omega_{j}$ is $f\left(\omega_{j}\right)=\gamma\left(\exp \left\{-i \omega_{j}\right\}\right) /(2 \pi)=\sigma^{2} q_{j} /(2 \pi)$. It follows that

$$
E\left(\frac{T}{2} \rho_{y j}^{2}\right)=2 \pi f\left(\omega_{j}\right) \quad \text { and } \quad V\left(\frac{T}{2} \rho_{y j}^{2}\right)=(4 \pi)^{2} f^{2}\left(\omega_{j}\right) \quad \text { if } \quad 0<\omega_{j}<\pi .
$$

Also, at zero frequency there is

$$
E\left(T \bar{y}^{2}\right)=2 \pi f(0) \quad \text { and } \quad V\left(T \bar{y}^{2}\right)=2(2 \pi)^{2} f^{2}(0),
$$

whereas, if $T=2 n$ is even, then, at the highest observable frequency, there is

$$
E\left(T \alpha_{n}^{2}\right)=2 \pi f\left(\omega_{n}\right) \quad \text { and } \quad V\left(T \alpha_{n}^{2}\right)=2(2 \pi)^{2} f^{2}\left(\omega_{n}\right) \quad \text { where } \quad \omega_{n}=\pi .
$$

There remains the question of the validity of approximating a linear ARMA process by a circular process. In the case of a white noise process, there is no 


\section{D.S.G. POLLOCK: Circulant Matrices}

effective distinction between a linear process and a circular process - the results for the periodogram analysis are identical.

In the case of an ordinary linear ARMA process, the generic element is $y_{t}=\sum_{j} \phi_{j} \varepsilon_{t-j}$, whereas, for a circular process, it is $y_{t}=\sum_{j} \phi_{j} \varepsilon_{(t-j \bmod T)}$. The difference lies entirely in the replacement of the presample elements in the sequence $\left\{\varepsilon_{t} ; t=0, \pm 1, \pm 2, \ldots\right\}$ by elements from within the sample.

It is clear that, if the sequence $\left\{\phi_{j} ; j=1,2, \ldots\right\}$ of ARMA parameters is absolutely convergent, then the presample values of $\left\{\varepsilon_{t}\right\}$ will be of diminishing significance in the formation of $y_{t}$ as they recede in time. On this basis, it is to be expected that, as the sample index $t$ increases, the corresponding elements of the two processes will converge. Therefore, as the sample size $T$ increases, the preponderance of the sample elements from the two process will converge, and this should lead to the convergence of their periodograms.

The formal proposition which expresses these notions is as follows:

Let $y(t)=\phi(L) \varepsilon(t)$, where $\varepsilon(t)$ is a white-noise process such that $E\left(\varepsilon_{t}\right)=0, V\left(\varepsilon_{t}\right)=\sigma^{2}$ for all $t$, and where the coefficients of the operator $\phi(L)$ are absolutely summable such that $\sum_{j=0}^{\infty}\left|\phi_{j}\right|<\infty$. Let $q_{j}=|\phi(z)|^{2}$ with $z=\exp \left\{-i \omega_{j}\right\}$. Then the periodogram of $y(t)$, based on $T$ observations, can be represented, at the Fourier frequency $\omega_{j}$, by

$$
\frac{T}{2} \rho_{y j}^{2}=\frac{T}{2}\left|q_{j}\right|^{2} \rho_{\varepsilon j}^{2}+R_{j},
$$

where $E\left(R_{j}\right) \rightarrow 0$ and $V\left(R_{j}\right) \rightarrow 0$ as $T \rightarrow \infty$.

Proofs of the proposition can be found, inter alia, in the texts of Priestley [11], Brockwell and Davies [3] and Fuller [5]. However, these proofs make no explicit reference to the circulant process.

It appears from (80) that, when they are scaled by a factor of $1 /(2 \pi)$, the ordinates of the periodogram of a circular process are unbiased estimates of the corresponding ordinates of the spectral density function. It also appears that the variances of these estimates do not diminish as the sample size $T$ increases. The same is roughly true of the ordinates of the periodogram of a linear process for which the results of (80) are asymptotically valid. The upshot is that the raw periodogram is not a consistent estimator of the spectral density function.

Nevertheless, as the sample size increases, the points at which the periodogram is defined become increasingly dense in every fixed frequency interval. Therefore, one can generate ever-improving estimates of the underlying spectral density function by averaging an increasing number of adjacent periodogram ordinates from within an ever-decreasing frequency interval.

Let $M=U \mu(D) \bar{U}$ be a circulant smoothing matrix which serves as an averaging operator in respect of the periodogram, and let $g(D)=U C \bar{U}$ be the diagonal matrix of (54) whose elements give rise to the periodogram ordinates. Then $s=M g(D) i$ is a vector whose elements are the ordinates of an estimated spectral density function based on local averages of the periodogram. 


\section{D.S.G. POLLOCK: Circulant Matrices}

An alternative expression for the vector of spectral estimates is $s=U \mu(D) G e_{0}$. This indicates that the estimated spectrum may be obtained by applying the relevant weights, contained within the diagonal matrix $\mu(D)$, to the vector $g=G e_{0}$ of the circular autocovariances and then applying a Fourier transform to the product.

The equivalence of the two expressions is demonstrated by confirming the following identities:

$$
\begin{aligned}
M g(D) i & =U \mu(D) \bar{U} g(D) i \\
& =U \mu(D) \bar{U} U G \bar{U} i \\
& =T^{1 / 2} U \mu(D) G e_{0} .
\end{aligned}
$$

\section{Testing the Homogeneity of a Time Series}

The purpose of this final section is to show how the results of the foregoing sections can be used in a simple way to construct statistical tests of hypotheses relating to the periodogram which are of a strictly classical nature.

Our specific object is to determine whether two adjacent segments of a time series have been generated by the same invariant process. Under the null hypothesis that the process is invariant, we may estimate a time-series model which can be used in constructing a filter for the purpose of reducing the series to white noise. Thereafter, we can test the null hypothesis that the two adjacent segments are generated by the same white-noise process.

An alternative to filtering the data is to divide the ordinates of the periodogram of the data by (estimates of) the corresponding spectral ordinates, in the manner of (77). The estimates of the spectral ordinates may be based upon the parameters of a fitted ARMA model. In that case, we should be taking the route to the spectrum which has been pursued in the example following (28). Alternatively, the spectral ordinates may be estimated by taking local averages of the periodogram ordinates in the manner indicated at the end of the previous section.

We shall avoid the unnecessary encumbrance of a notation which indicates explicitly that the vectors $y_{1}$ and $y_{2}$, which are from a putative white-noise process, have been obtained by filtering the data. Therefore, according to the null hypothesis, $y_{1} \sim N\left(0, \sigma^{2} I_{N}\right)$ and $y_{2} \sim N\left(0, \sigma^{2} I_{N}\right)$ are two adjacent segments both comprising $N$ elements, which are generated by a white-noise process which has a variance of $\sigma^{2}$. Then the corresponding Fourier transforms can be calculated for both segments independently via a scheme which is represented by the equation

$$
\left[\begin{array}{l}
\zeta_{1} \\
\zeta_{2}
\end{array}\right]=\left[\begin{array}{cc}
U & 0 \\
0 & U
\end{array}\right]\left[\begin{array}{l}
y_{1} \\
y_{2}
\end{array}\right]
$$

According to the hypothesis, $\bar{\zeta}_{1}^{\prime} \zeta_{1} / \sigma^{2} \sim \chi^{2}(N)$ and $\bar{\zeta}_{2}^{\prime} \zeta_{2} / \sigma^{2} \sim \chi^{2}(N)$ are mutually independent chi-square variates and their sum $\left(\bar{\zeta}_{1}^{\prime} \zeta_{1}+\bar{\zeta}_{2}^{\prime} \zeta_{2}\right) / \sigma^{2} \sim \chi^{2}(2 N)$ is also a chi-square variate. 


\section{D.S.G. POLLOCK: Circulant Matrices}

Now consider forming the vector

$$
\xi=\frac{1}{\sqrt{2}}\left[\begin{array}{ll}
U & U
\end{array}\right]\left[\begin{array}{l}
y_{1} \\
y_{2}
\end{array}\right] .
$$

We can show easily that $\bar{\xi}^{\prime} \xi / \sigma^{2} \sim \chi^{2}(N)$ is a chi-square variate. Moreover, By invoking Cochrane's theorem, it can be show that, under the null hypothesis,

$$
\frac{\bar{\xi}^{\prime} \xi}{\sigma^{2}} \sim \chi^{2}(N) \quad \text { and } \quad \frac{\bar{\zeta}_{1}^{\prime} \zeta_{1}+\bar{\zeta}_{2}^{\prime} \zeta_{2}-\bar{\xi}^{\prime} \xi}{\sigma^{2}} \sim \chi^{2}(N)
$$

are mutually independent chi-square variates. Their ratio is therefore an $F(N, N)$ variate; and this statistic provides the means of testing the hypothesis that $y_{1}$ and $y_{2}$ have been generated by the same process.

The relevant version of Cochrane's theorem is as follows:

Let $y \sim N\left(0, \sigma^{2} I_{2 N}\right)$, and let $P_{1}+P_{2}=I_{2 N}$ be a sum of two matrices which are symmetric and idempotent with $\operatorname{rank}\left(P_{1}\right)=$ $\operatorname{rank}\left(P_{2}\right)=N$ such that $P_{i}=P_{i}^{2}$ and $P_{i} P_{j}=0$ when $i \neq j$. Then $y^{\prime} P_{i} y \sim \chi^{2}(N) ; i=1,2$ are independent chi-square variates such that $\left(y^{\prime} P_{1} y+y^{\prime} P_{2} y\right) / \sigma^{2}=y^{\prime} y / \sigma^{2} \sim \chi^{2}(2 N)$.

To confirm that these conditions are fulfilled, we note that $U$ is a unitary matrix such that $\bar{U} U=I$, where $\bar{U}$ is the conjugate transpose of $U$, and we note that

$$
P_{1}=\frac{1}{2}\left[\begin{array}{l}
\bar{U} \\
\bar{U}
\end{array}\right]\left[\begin{array}{ll}
U & U
\end{array}\right]=\frac{1}{2}\left[\begin{array}{ll}
I & I \\
I & I
\end{array}\right]
$$

is a symmetric idempotent matrix. We define $P_{2}=I-P_{1}$ and we note that $P_{1} P_{2}=0$. It is easy to see in reference to (85) that

$$
\begin{aligned}
\frac{1}{\sigma^{2}}\left(\bar{\zeta}_{1}^{\prime} \zeta_{1}+\bar{\zeta}_{2}^{\prime} \zeta_{2}\right) & =\frac{1}{\sigma^{2}}\left(y_{1}^{\prime} \bar{U} U y_{1}+y_{2}^{\prime} \bar{U} U y_{2}\right) \\
& =\frac{1}{\sigma^{2}} y^{\prime} y \sim \chi^{2}(2 N) .
\end{aligned}
$$

Now we see, in view of the properties of $P_{1}$ and $P_{2}$, that

$$
\frac{\bar{\xi}^{\prime} \xi}{\sigma^{2}}=\frac{y^{\prime} P_{1} y}{\sigma^{2}} \quad \text { and } \quad \frac{\bar{\zeta}_{1}^{\prime} \zeta_{1}+\bar{\zeta}_{2}^{\prime} \zeta_{2}-\bar{\xi}^{\prime} \xi}{\sigma^{2}}=\frac{y^{\prime} P_{2} y}{\sigma^{2}}
$$

are mutually independent chi-square variates of $N$ degrees of freedom each.

The $F$ statistic, which is formed from the ratio of the chi-square variates of (87), provides an overall test of the hypothesis of homogeneity. It will serve to detect cases where the power or variance differs between the two data segments. However, the statistic will not detect changes in the spectral structure of the process that do not affect the overall power. 


\section{D.S.G. POLLOCK: Circulant Matrices}

Changes in the distribution of the power can be detected by making comparisons of the periodograms of the adjacent data segments in a number of disjoint frequency bands. The relevant statistics may be obtained from the existing $F$ statistic by breaking the sum in the numerator into parts which constitute mutually independent chi-square variates.

Let $S$ be a selection matrix of order $T \times T$ with zeros and units on the principal diagonal and with zeros elsewhere. Let the matrix be subject to the condition that, if it contains a unit in the $j$ th position, then it also has a unit in the position $T-j$. If, in addition, the units are contiguous within two separate blocks, then the matrix will serve to select from the vectors $\zeta_{1}, \zeta_{2}$ and $\xi$ all of the periodogram ordinates which fall in a specific frequency band.

By applying the selection matrix to equations (85) we get

$$
\left[\begin{array}{l}
S \zeta_{1} \\
S \zeta_{2}
\end{array}\right]=\left[\begin{array}{cc}
S U & 0 \\
0 & S U
\end{array}\right]\left[\begin{array}{l}
y_{1} \\
y_{2}
\end{array}\right]
$$

By applying the selection matrix to equations (86), we get

$$
S \xi=\frac{1}{\sqrt{2}}\left[\begin{array}{ll}
S U & S U
\end{array}\right]\left[\begin{array}{l}
y_{1} \\
y_{2}
\end{array}\right]
$$

Let $S \zeta_{1}=\zeta_{S 1}, S \zeta_{2}=\zeta_{S 2}$ and $S \xi=\xi_{S}$. Then, under the hypothesis that there are no changes in the spectral density function within the selected range of frequencies, we should have

$$
\frac{\bar{\zeta}_{S 1}^{\prime} \zeta_{S 1}+\bar{\zeta}_{S 2}^{\prime} \zeta_{S 2}-\bar{\xi}_{S}^{\prime} \xi_{S}}{\bar{\xi}^{\prime} \xi} \sim F(q, N), \quad \text { where } \quad q=\operatorname{rank}(S)
$$

To establish this result, it is enough to show that the numerator and the denominator of the statistic are quadratic forms in the same spherical normal vector which are based on mutually orthogonal symmetric idempotent matrices with ranks that correspond to the degrees of freedom of the $F$ distribution.

The quadratic form of the denominator, which can be expressed as $y^{\prime} P_{1} y$ where $y \sim N\left(0, \sigma^{2} I_{N}\right)$, is based on the symmetric idempotent matrix $P_{1}=P_{1}^{\prime}=$ $P_{1}^{2}$ of (89) which has $\operatorname{rank}\left(P_{1}\right)=N$. The quadratic form of the numerator, which can be expressed as $y^{\prime} P_{S} y$, is based on the matrix

$$
P_{S}=\frac{1}{2}\left[\begin{array}{cc}
\bar{U} S U & -\bar{U} S U \\
-\bar{U} S U & \bar{U} S U
\end{array}\right]
$$

It can be seen that $P_{S}=P_{S}^{\prime}=P_{S}^{2}$ and that $P_{1} P_{S}=0$. Moreover, $\operatorname{rank}\left(P_{S}\right)=$ $\operatorname{rank}(S)=q$. Thus the conditions in question are fulfilled. 


\section{D.S.G. POLLOCK: Circulant Matrices}

\section{References}

[1] Anderson, T.W., (1971), The Statistical Analysis of Time Series, John Wiley and Sons, Chichester.

[2] Catalan, E., (1846), Récherches sur les Déterminants, Bulletin de l'Academie Royale de Belgique, 13, 534-555.

[3] Brockwell, P.J., and R.A. Davis, (1987), Time Series: Theory and Methods, Springer Verlag, New York.

[4] Davis, P.J., (1979), Circulant Matrices, John Wiley and Sons, New York.

[5] Fuller, W.A., (1976), Introduction to Statistical Time Series, John Wiley and Sons, New York.

[6] Muir, T., (1906), The Theory of Circulants in the Historical Order of Development up to 1860, Proceedings of the Royal Society of Edinburgh, 26, 390-398.

[7] Muir, T., (1911), The Theory of Circulants from 1861 to 1880, Proceedings of the Royal Society of Edinburgh, 32, 136-149.

[8] Muir, T., (1915), The Theory of Circulants from 1880 to 1900, Proceedings of the Royal Society of Edinburgh, 36, 151-179.

[9] Muir, T., (1923), The Theory of Circulants from 1900 to 1920, Proceedings of the Royal Society of Edinburgh, 44, 218-241.

[10] Pollock, D.S.G., (1993), On the Criterion Function for ARMA Estimation, Journal of Statistical Inference and Planning, 36, 413-430.

[11] Priestley, M.B., (1981), Spectral Analysis and Time Series, Academic Press, London.

[12] Schlesinger, M.E. and N. Ramankutty, (1994), An Oscillation in the Global Climate System of 65-70 years, Nature, 377, 723-726.

[13] Vautard, R. and M. Ghil, (1989), Singular Spectral Analysis in Nonlinear Dynamics with Applications to Paleoclimatic Time Series, Physica, 3D, 395-424. 


\section{Q Queen Mary \\ University of London}

This working paper has been produced by the Department of Economics at Queen Mary, University of London

Copyright $\odot 2000$ Stephen Pollock All rights reserved.

\section{Department of Economics}

Queen Mary, University of London

Mile End Road

London E1 4NS

Tel: +44 (0)20 78825096 or Fax: +44 (0)20 89833580

Email: j.conner@qmw.ac.uk

Website: www.econ.qmw.ac.uk/papers/wp.htm 